\title{
A unique role for p53 in the regulation of M2 macrophage polarization
}

\author{
L Li, ${ }^{* 1}$, DSW Ng ${ }^{2}$, W-C Mah ${ }^{3}$, FF Almeida ${ }^{4}$, SA Rahmat ${ }^{1}$, VK Rao ${ }^{5}$, SC Leow ${ }^{6}$, F Laudisi ${ }^{4}$, MT Peh ${ }^{2}$, AM Goh ${ }^{1}$, JSY Lim $^{7}$, GD Wright ${ }^{7}$, \\ A Mortellaro ${ }^{4}$, R Taneja ${ }^{5}$, F Ginhoux ${ }^{4}$, CG Lee ${ }^{3,8}$, PK Moore ${ }^{2}$ and DP Lane ${ }^{*, 1}$
}

P53 is critically important in preventing oncogenesis but its role in inflammation in general and in the function of inflammatory macrophages in particular is not clear. Here, we show that bone marrow-derived macrophages exhibit endogenous p53 activity, which is increased when macrophages are polarized to the M2 (alternatively activated macrophage) subtype. This leads to reduced expression of M2 genes. Nutlin-3a, which destabilizes the p53/MDM2 (mouse double minute 2 homolog) complex, promotes p53 activation and further downregulates M2 gene expression. In contrast, increased expression of M2 genes was apparent in M2polarized macrophages from p53-deficient and p53 mutant mice. Furthermore, we show, in mice, that p53 also regulates M2 polarization in peritoneal macrophages from interleukin-4-challenged animals and that nutlin-3a retards the development of tolerance to Escherichia coli lipopolysaccharide. P53 acts via transcriptional repression of expression of c-Myc (v-myc avian myelocytomatosis viral oncogene homolog) gene by directly associating with its promoter. These data establish a role for the p53/MDM2/c-MYC axis as a physiological 'brake' to the M2 polarization process. This work reveals a hitherto unknown role for $\mathrm{p} 53$ in macrophages, provides further insight into the complexities of macrophage plasticity and raises the possibility that p53-activating drugs, many of which are currently being trialled clinically, may have unforeseen effects on macrophage function. Cell Death and Differentiation (2015) 22, 1081-1093; doi:10.1038/cdd.2014.212; published online 19 December 2014

Macrophages have key roles in the response to stress, injury, infection and inflammation. The M1 (classically activated macrophages) are induced by lipopolysaccharide (LPS) and cytokines such as interferon- $\gamma$ (IFN $\gamma$ ) and characterized by the expression of a wide range of proinflammatory genes. M2 (alternatively activated macrophages) are induced by T helper type 2 (Th2) cytokines such as interleukin-4 (IL4) and IL13 and express high levels of anti-inflammatory and tissue repair marker genes. M2 macrophages perform immunoregulatory functions including defense against infection, promotion of angiogenesis and wound healing. ${ }^{1}$

Macrophages exist on a continuum between M1 and M2 subtypes undergoing dynamic changes between these different functional states depending on changes in their microenvironment. This 'plasticity' involves extensive changes in macrophage gene sets and provides the potential to develop drugs to manipulate the macrophage subtype. ${ }^{2}$
As such, macrophage polarization, and its molecular basis, has been vigorously researched in recent years.

P53 has a crucial role in cancer by controlling the expression of genes involved in apoptosis, cell cycle arrest, metabolism and DNA repair. ${ }^{3}$ While inflammation is increasingly recognized as a factor in determining the predisposition to cancer, ${ }^{4}$ the role of $p 53$ in inflammation is not clear. Macrophages from $\mathrm{p} 53^{-/-}$mice produce increased quantities of proinflammatory cytokines in response to LPS, ${ }^{5}$ while peritoneal macrophage count and susceptibility to lethal septic shock are increased in $\mathrm{p}^{-1-}$ mice administered LPS. ${ }^{6}$ Although a role for $\mathrm{p} 53$ in $\mathrm{M} 1$ macrophage function has been suggested, there is little information relating to its effect in M2 macrophages. We show here that p53 is important for IL4/ IL13-activated M2 macrophage polarization and that this is largely due to repression of transcription of c-MYC (v-myc avian myelocytomatosis viral oncogene homolog) and a subset of its regulated genes.

\footnotetext{
${ }^{1}$ p53 Laboratory, A*Star, 8A Biomedical Grove, Immunos, Singapore 138648; ${ }^{2}$ Neurobiology Program, Life Science Institute and Department of Pharmacology, Yong Loo Lin School of Medicine, National University of Singapore, Singapore; ${ }^{3}$ Division of Medical Sciences, National Cancer Centre, Singapore and NUS Graduate School for Integrative Sciences and Engineering, National University of Singapore, Singapore; ${ }^{4}$ Singapore Immunology Network, $A^{\star}$ Star, Singapore; ${ }^{5}$ Department of Physiology, Yong Loo Lin School of Medicine, National University of Singapore, Singapore; ${ }^{6}$ Singapore Institute of Clinical Sciences, $A^{*}$ Star, Singapore; ${ }^{7}$ Microscopy Unit, Institute of Medical Biology, $A^{*}$ Star, Singapore and ${ }^{8}$ Department of Biochemistry, Yong Loo Lin School of Medicine, National University of Singapore and Cancer and Stem Cell Biology Program, DUKE-NUS Graduate Medical School, Singapore

*Corresponding authors: L Li or DP Lane, p53 Laboratory, A*Star, 8A Biomedical Grove, \#06-06, Immunos, Singapore 138648. Tel: +65 6407 0673; E-mail: lli@p53Lab. a-star.edu.sg or dplane@p53Lab.a-star.edu.sg

Abbreviations: AKT, protein kinase B; Arg1, arginase-1; ATP, adenosine triphosphate; BMDM, bone marrow-derived macrophage; ChIP, chromatin immunoprecipitation; C-MYC, v-myc avian myelocytomatosis viral oncogene homolog; CREB, CAMP response element-binding protein; DECTIN-1, C-type lectin domain family 7 member A/Dectin; DMSO, dimethylsulfoxide; CXCL9, C-X-C motif chemokine 9; CXCL10, C-X-C motif chemokine 10; EGFP, enhanced green fluorescent protein; IFN $\gamma$, interferon- $\gamma$; IL4, interleukin-4; IL4C, slow-releasing interleukin-4; IL12p40, the p40 (40 kDa) subunit of interleukin-12; IL6, interleukin-6; IL13, interleukin-13; IL13R $\alpha$ 1, interleukin-13 receptor $\alpha$ 1; IRF4, interferon regulatory factor 4; IRF5, interferon regulatory factor 5; LPS, lipopolysaccharide; M1, classically activated macrophage; M2, alternatively activated macrophage; M-CSF, macrophage colony-stimulating factor; MDM2, mouse double minute 2 homolog; NF- $\kappa$ B, nuclear factor $\kappa \mathrm{B}$; PI3K, phosphatidylinositol 3-kinase; RT-qPCR, reverse transcription-quantitative PCR; STAT1, signal transducer and activator of transcription 1; STAT6, signal transducer and activator of transcription 6; TAM, tumor-associated macrophage; TH, T helper; TNF $\alpha$, tumor necrosis factor $\alpha$

Received 31.7.14; revised 14.11.14; accepted 17.11.14; Edited by S Piccolo; published online 19.12.14
} 


\section{Results}

Polarization of macrophages activates p53. Polarization of macrophages to the M1 or M2 subtype increased the expression of p53 and its downstream markers including MDM2 (mouse double minute 2 homolog) and p21 (Figure 1a). P53 transcriptional activity also increased in both M1- and M2-polarized macrophages from transgenic mice carrying a p53-responsive enhanced green fluorescent protein (EGFP) reporter construct (Figures $1 a$ and b). Activation of p53 was more prominent in M1-polarized macrophages but was still present in M2-polarized cells and was potentiated by nutlin-3a, which inhibits the p53-MDM2 interaction (Figure 1a). Early (15 min) phosphorylation of AKT (protein kinase B) at Ser473 followed (30 min onwards) by phosphorylation of MDM2 at Ser166 (Figure 1c) occurred during M2 polarization. The phosphatidylinositol 3kinase (PI3K) inhibitor, LY294002, abolished AKT and MDM2 phosphorylation in M2-polarized macrophages
(Figure 1d). Therefore, M2 polarization activates PI3K and AKT, which phosphorylates MDM2 and increases p53 ubiquitination, which was detectable in the presence of the proteasome inhibitor, MG132 (Figure 1e). Nutlin-3a, but not nutlin-3b (the less active enantiomer; Elsawy et al. ${ }^{7}$ ), significantly reduced p53 ubiquitination (Figure 1e), confirming a role for MDM2 in this process, whereas LY294002 partially blocked p53 ubiquitination, indicating an important, but more upstream, role of PI3K/AKT activation. In contrast, minimal ubiquitination of p53 occurred in M1 macrophages and this was largely unaffected by nutlin-3a, nutlin-3b or LY294002 (Figure 1e), although LY294002 treatment of macrophages in the presence of MG132 did result in some degree of cell death (data not shown). We propose that these molecular events increase p53 turnover and underpin the low, but greater than basal, level of p53-mediated transcriptional activity in M2-polarized macrophages.

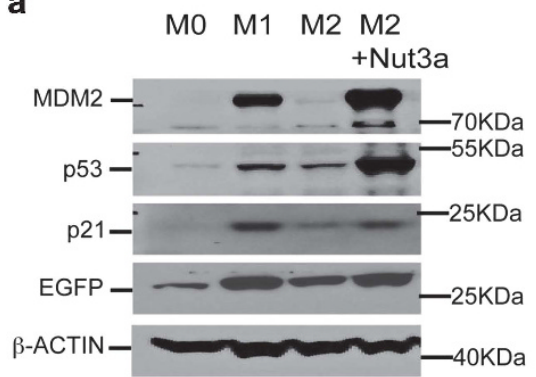

C

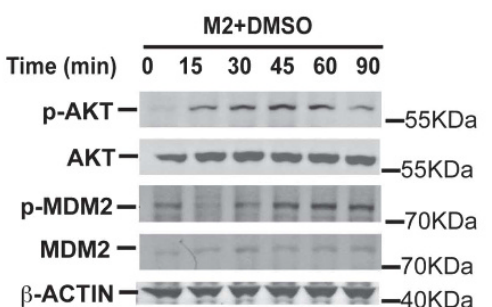

d

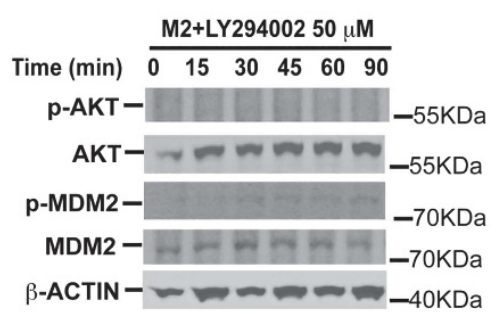

b
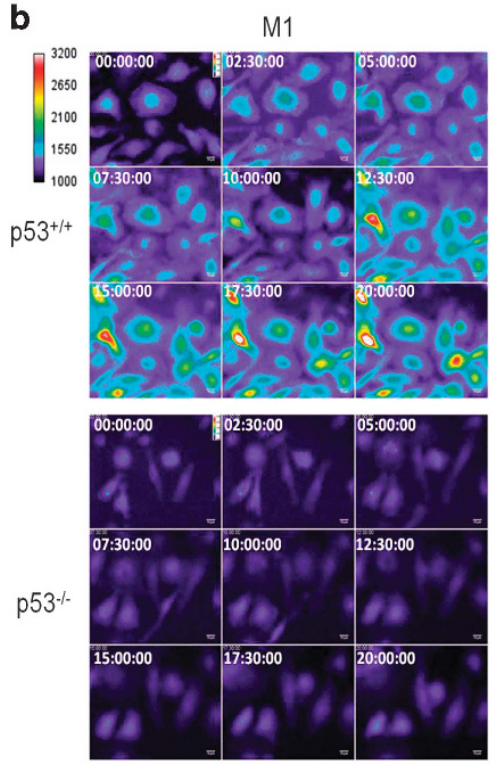

$\mathrm{M} 2$
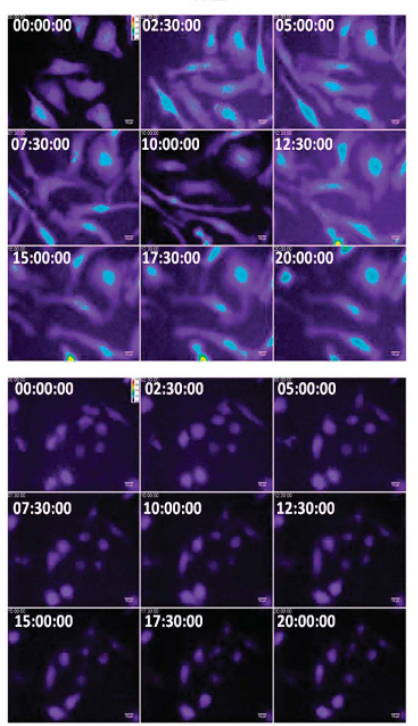

e

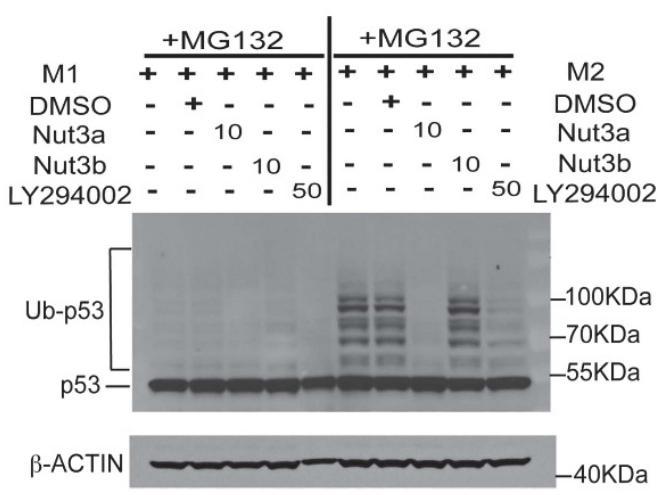

Figure 1 Effect of macrophage polarization on p53 activation. (a) Expression of p53 and downstream proteins, as well as expression of EGFP in M0, M1 and M2 macrophages and M2 macrophages treated with nutlin (Nut)-3a (10 $\mu \mathrm{M})$ from p53 reporter mice as determined by western blotting at $24 \mathrm{~h}$ with $\beta$-ACTIN as a loading control. Results are representative of three independent experiments. (b) Individual frames showing change in EGFP fluorescence following live-cell imaging of M1- and M2-polarized macrophages obtained from $p 53^{+/+}$and $p 53^{-1-}$ mice at time zero and a further eight time points up to $20 \mathrm{~h}$. Data are representative of four independent experiments. (c and $\mathbf{d}$ ) Time-dependent phosphorylation of AKT at Ser473 and MDM2 at Ser166 in M2-polarized macrophages pre-treated (30 min) with either DMSO (c) or the PI3K inhibitor LY294002 $(\mathrm{d} ; 50 \mu \mathrm{M})$. Total AKT and MDM2 expression together with $\beta$-ACTIN as a loading control over the same time period are shown. Data are representative of three independent experiments. (e) Polyubiquitination of endogenous p53 in M1- (left panel) and M2- (right panel) polarized macrophages in the presence of the proteasome inhibitor MG132 $(10 \mu \mathrm{M})$ combined with Nut3a $(10 \mu \mathrm{M})$, Nut3b $(10 \mu \mathrm{M})$, LY294002 $(50 \mu \mathrm{M})$ or vehicle (DMSO). Results show an individual experiment with $\beta$-ACTIN as a loading control and are representative of three independent experiments 
P53 regulates polarization of M2 macrophages in vitro. The role of $\mathrm{p} 53$ in $\mathrm{M} 2$ polarization was studied using nutlin-3a, which enhanced p53 activity in macrophages as evidenced by the time- and concentration-dependent upregulation of two key transcriptional target genes, p21 and Mdm2 (Supplementary Figures S1A and B). The threshold effective concentration of nutlin-3a was $10 \mu \mathrm{M}$, which did not affect cell viability as determined by measurement of cellular ATP (adenosine triphosphate) (Supplementary Figure S1C).

Specific genes regulated by activation of p53 in M2 macrophages were scrutinized by whole genome microarray. A set of M2 genes was identified, which were inversely affected by nutlin-3a (Figure 2a). Among these, nutlin-3a reduced the expression of Irf4 (interferon regulatory factor 4), c-Myc, Arg1 (arginase-1) and Retnla (Fizz1) (Figures 2b-e). PM2 and M011, stapled peptide inhibitors of the p53-MDM2 interaction (Supplementary Figure S1D; Wei et al. ${ }^{8}$ ), were also active (Supplementary Figures S1E and F). Nutlin-3b did not activate p53 or repress M2 activation (Supplementary Figure S1E). Moreover, nutlin-3a did not affect the expression of $\mathrm{M} 2$ markers in macrophages from $\mathrm{p} 53^{-1-}$ animals (Supplementary Figure S1G), thereby confirming that this effect is p53-dependent.

The ability of nutlin-3a to alter the expression of classical M1 marker genes in M1 macrophages was also assessed (Supplementary Figure S2). Expression of Nos2, I/12p40 (the p40 (40 kDa) subunit of interleukin-12), Cxcl9 (C-X-C motif chemokine 9) and $\mathrm{CxCl} 10$ (C-X-C motif chemokine 10) was reduced by nutlin-3a. There was minimal expression of these markers in nutlin-3a-treated M2 cells (Supplementary Figure S2A). Comparing the whole genome transcriptional profile of cells polarized to the $\mathrm{M} 1, \mathrm{M} 2$ or $\mathrm{M} 2$ treated with nutlin-3a subtypes, other M1 genes upregulated by nutlin-3a in M2-polarized cells were identified (Supplementary Figure S2B). Mapping these genes using ingenuity pathway analysis revealed that many were regulated by proinflammatory LPS and TNFa (tumor necrosis factor-a) (Supplementary Table S1). We conclude that activation of p53 by nutlin-3a in M2 macrophages may result in a macrophage phenotype intermediate between $\mathrm{M} 1$ and $\mathrm{M} 2$.

The proportion of $\mathrm{M} 1$ and $\mathrm{M} 2$ macrophages carrying specific M1 (CD80) or M2 (CD206 and DECTIN-1 (C-type lectin domain family 7 member A/Dectin)) cell surface markers was assessed by FACS analysis (Figure 3a). The majority of M2-polarized macrophages were CD206 ${ }^{\text {high }} /$ DECTIN-1 ${ }^{\text {high }}$ / $\mathrm{CD}^{\text {low }}$ consistent with an M2 subtype, while M1-polarized macrophages were largely CD80 ${ }^{\text {high }} /$ CD206 ${ }^{\text {low }} / D E C T I N-1^{\text {low }}$. Activation of p53 with nutlin-3a shifted the M2 macrophage population away from this classical M2 subtype towards a CD80 high $/$ CD206 $^{\text {low }} / D E C T I N-1^{\text {low }}$ subtype more closely resembling the M1 subtype (Figure $3 a$ ) but did not affect M1 macrophages, which remained $\mathrm{CD} 80^{\text {high/ }}$ CD206 $6^{\text {low/DECTIN-1 }} 1^{\text {low }}$. Hence, we suggest that nutlin-3ainduced activation of p53 under M2-polarizing conditions thwarts the establishment of the M2 subtype.

The functional significance of p53 in M2 macrophages was also studied. Nutlin-3a reduced the expression of Arg1 (Figures $2 \mathrm{~d}$ and $\mathrm{f}$ ) and inhibited its catalytic activity in M2 macrophages (Figure $3 b$ ). Nutlin-3a also reduced IL4-induced M2 macrophage proliferation (Figures $3 c$ and d). Thus, nutlin-3a impairs not only $\mathrm{M} 2$ gene expression but also the functional consequences of M2 activation.

The effect of endogenous p53 activation on M2 gene

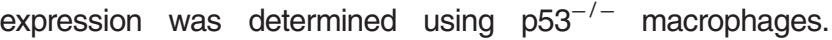
Loss of p53 increased the expression of several M2 genes (Figures $4 a-d$ ), increased arginase activity (Figure 4e) and enhanced proliferation of M2 macrophages (Figures $4 \mathrm{f}$ and $\mathrm{g}$ ). In control experiments, no difference between $\mathrm{p} 53^{+/+}$and $\mathrm{p} 53^{-/-}$ mice with respect to, (i) rate of M-CSF (macrophage colonystimulating factor)-induced differentiation of bone marrow cells into $\mathrm{F} 4 / 80^{+}$macrophages (Supplementary Figure S3A), (ii) expression of receptor genes for LPS (i.e. toll-like receptor-4, TIr4; Supplementary Figure S3B), IFN $\gamma$ (Ifnyr1 (interferon- $\gamma$ receptor 1); Supplementary Figure S3C), M-CSF (Csf1ra (colony-stimulating factor 1 receptor $a$ ); Supplementary Figure S3D), IL4 (IL4ra (interleukin-4 receptor a); Supplementary Figure S3E) or IL13 receptors (I/13ra 1; Supplementary Figure S3F) or (iii) cellular ATP content (Supplementary Figure S3G) was noted. Any change in M2 macrophage gene expression in p53-deficient cells is unrelated to differences in the effectiveness of the inflammatory stimuli used to polarize these cells or to defective macrophage development or aberrant cell death.

Expression of p53 and its downstream targets in wild-type M2-polarized macrophages was compared with that of knockin animals carrying either one or two copies of a DNA-binding mutant ( $p 53^{R 172 H}$, equivalent to the $p 53^{R 175 H}$ hotspot mutation in human cancers). P53 accumulation was more abundant in mutant cells regardless of their polarization state. Mutant cells were also refractory to nutlin-3a-evoked p53 stabilization (Figure 4h). Moreover, while p53 accumulated in mutant cells, it did not cause translation of downstream target proteins such as MDM2 and p21 or p53 activation as determined by EGFP expression (Figure 4h). Importantly, loss of p53 transactivation in mutant cells was associated with copy-dependent upregulation of the expression of several key M2 markers, including IRF4, c-MYC and FIZZ1 (Figure 4i). In addition, neither nutlin$3 a$ nor nutlin-3b reduced the expression of $M 2$ signature markers in mutant cells (Figure 4i). These findings show that endogenous p53 activity in M2-polarized macrophages, although low, is still sufficient to limit M2 gene expression and that this effect depends on its DNA-binding activity.

Molecular mechanism of action of p53 in M2 macrophages. The molecular mechanism of action of p53 in regulating M2 polarization is unknown. One potential target is p21, a well-characterized downstream effector of p53 activity, ${ }^{9}$ which is upregulated during M2 polarization. We found that p53 bound to the p21 promoter in M2-polarized macrophages and this was further enhanced by nutlin-3a (Supplementary Figure S4A). Expression of the M2 markers, IRF4 and FIZZ1, was increased in p21 $1^{-/-}$M2-polarized macrophages, while the expression of c-MYC was unchanged (Supplementary Figure S4B). Activation of p53 using nutlin-3, PM2 or M011 was equally effective in inhibiting the expression of M2 markers in both wild-type and $\mathrm{p}_{21} 1^{-1}$ cells (Supplementary Figure S4B). Although p21 regulates the expression of some M2 markers, its absence does not alter the effect of nutlin-3 on macrophage polarization. Hence, p21 is unlikely to have a major role in the effect of p53 activation in M2 polarization. Finally, proliferation of M2 
a

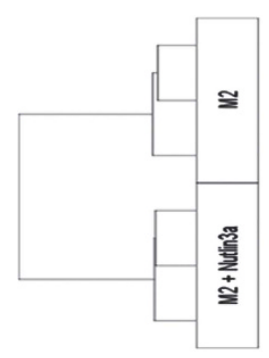

Hierarchical Clustering

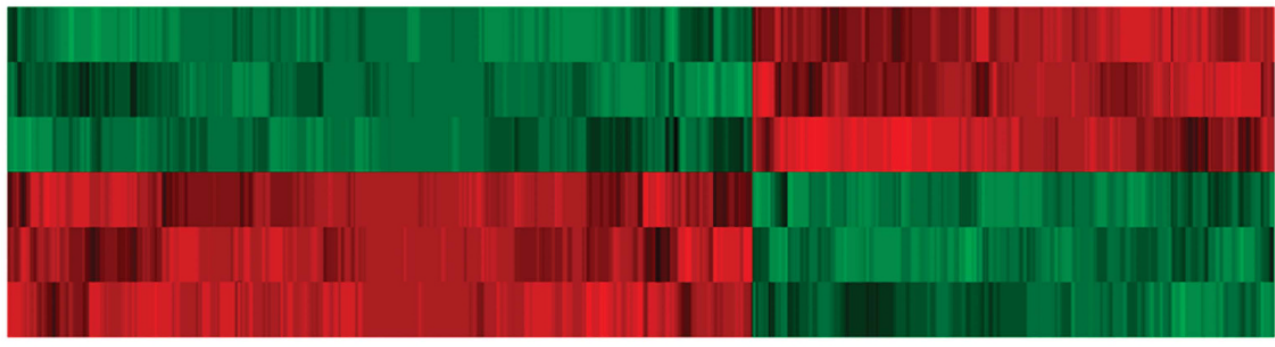

$\frac{\text { Scale }}{-2.00}$

b

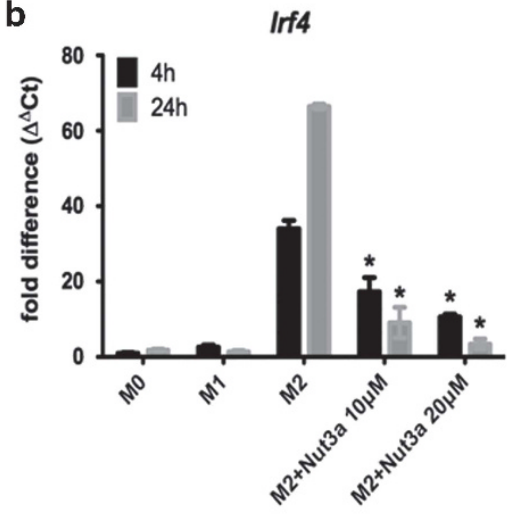

C

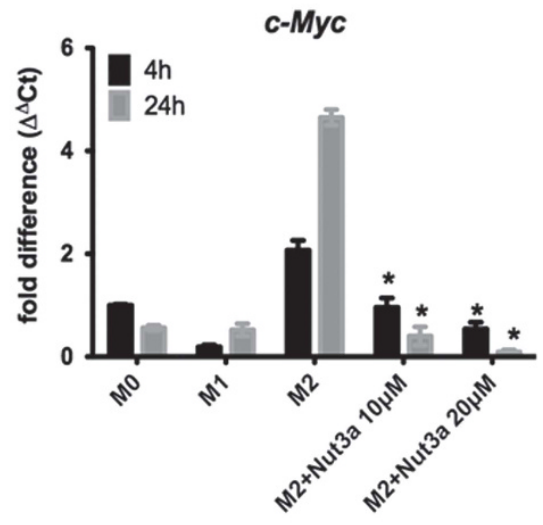

d

Arg1

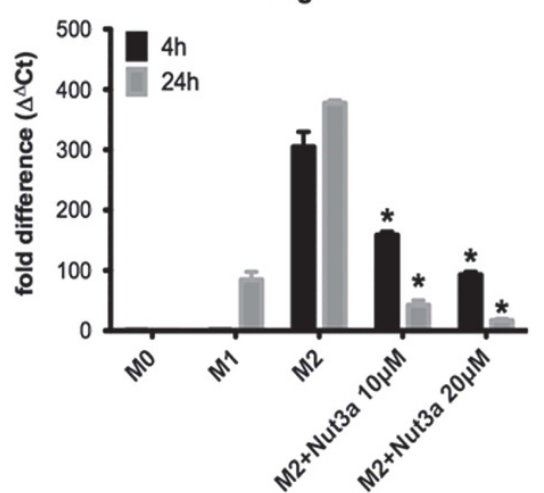

e

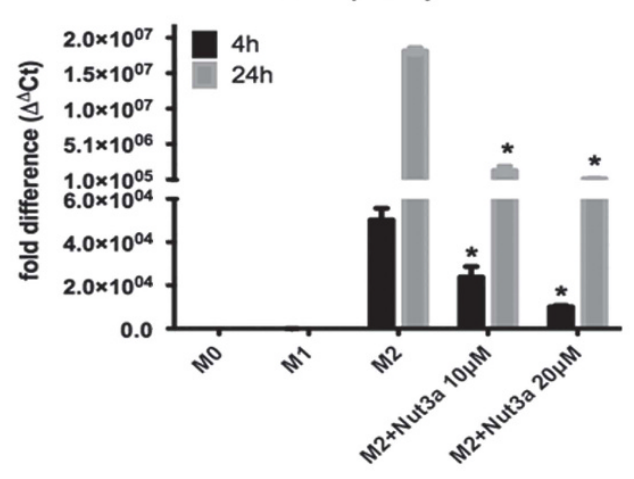

f DMSO $\frac{\text { M0 }}{+} \frac{\text { M1 }}{+} \frac{\text { M2 }}{+}$

Nut3a $(\mu \mathrm{M})$

n.s. -

C-MYC -

$-70 \mathrm{KDa}$

IRF4- $=-50 \mathrm{KDa}$

FIZZ1- - - $14 \mathrm{KDa}$

ARG1- $\quad \omega \pi-49 K D a$

p21- $-19 K D a$

$\beta$-ACTIN- $-39 \mathrm{KDa}$ 
(but not M1) cells from $\mathrm{p} 21^{-1-}$ mice was increased and this was not altered by nutlin-3a (Supplementary Figure S4C). It is therefore unlikely that $\mathrm{p} 53$ affects macrophage polarization indirectly by an effect on proliferation.

Another candidate is C-MYC, which was enriched in M2polarized macrophages and was proposed to regulate M2 macrophage activation. ${ }^{10,11}$ It was not clear whether induction of c-MYC in M2-polarized macrophages is a primary event in response to IL4/IL13 stimulation or secondary to STAT6 (signal transducer and activator of transcription 6) activation. To clarify, c-MYC expression in macrophages from wild-type and STAT6-deficient mice was compared. Loss of STAT6 abolished the augmented expression of c-MYC in M2 cells (Supplementary Figures S4A and B), indicating that induction of $\mathrm{c}-\mathrm{MYC}$ depends on STAT6. Expression of c-MYC was downregulated by nutlin-3a (Figures 2c, f and 4b, i). Based on global gene expression and ingenuity pathway analysis of M2 macrophages treated with nutlin-3a, c-MYC-regulated genes were significantly enriched, suggesting that the c-MYC pathway contributes to nutlin-3a-mediated inhibition of M2 gene transcription (Supplementary Table S2). Interestingly, a recent study analyzing RNA-seq-based high-resolution transcriptome data identified both c-MYC and p53 as major hubs in the human M2 macrophage network. ${ }^{12}$ We propose that c-MYC has a key role in the response of M2 macrophages to p53 activation.

Inhibition of c-MYC using 10058F4, which prevents transactivation of $\mathrm{c}-\mathrm{MYC}$ target gene expression, reduced the expression of M2 markers, including IRF4, FIZZ1 and c-MYC itself (Figure 5a and Supplementary Figure S5C) without affecting the expression of p53, MDM2 or p21 (Figure 5a and Supplementary Figures S5D-F). The effect of 10058F4 on global gene expression in M2-polarized cells closely resembled that of nutlin-3a producing a distinctive population of macrophages with a gene set intermediate between the M1 and M2 subtypes (Figure $5 \mathrm{~b}$ ). Both drugs suppressed a similar subset of M2 transcriptome activation (Figure 5c). By comparing differentially expressed gene sets in $\mathrm{M} 2$ cells from (i) $\mathrm{p} 53^{+/+}$, (ii) $\mathrm{p} 53^{+/+}$treated with nutlin-3a, (iii) $\mathrm{p} 53^{+/+}$treated with $10058 \mathrm{~F} 4$ and (iv) $\mathrm{p} 53^{-1-}$, a series of M2 genes, which were strictly p53-dependent and regulated by c-MYC, were identified (Figure 5c) and confirmed by RT-qPCR (Supplementary Figure S5C). Interestingly, 10058F4 reduced the expression of $\mathrm{M} 2$ markers in $\mathrm{p} 53^{\mathrm{R} 172 \mathrm{H} / \mathrm{R} 172 \mathrm{H}}$ macrophages, indicating that it does not require a functional p53 to be effective (Figure 4i). These data show that c-MYC and p53 reciprocally control an overlapping subset of M2-specific genes and, importantly, that c-MYC acts downstream from $\mathrm{p53}$.
The mechanism by which p53 negatively regulates $c-M y c$ was investigated by chromatin immunoprecipitation (ChIP) to monitor p53 binding to the $c-M y c$ promoter. Binding of p53 at the promoter regions of $c-M y c$ was present in $\mathrm{M} 2$-stimulated cells at all p53 binding sites examined (Figure 5d). Treatment of M2-polarized cells with nutlin-3a enhanced p53 recruitment to the $c-M y c$ promoter (Figure $5 \mathrm{~d}$ ). H3K9 acetylation, an epigenetic marker signifying transcriptionally active chromatin, at two loci associated with abundant p53 binding (P1 and $\mathrm{P} 2)$ in the $c-M y c$ promoter was also studied. As expected, significant enrichment of H3K9 acetylation occurred at both sites in M2-polarized macrophages and nutlin-3a markedly reduced acetylation in these cells (Figure $5 \mathrm{e}$ ) consistent with the ability of nutlin-3a to downregulate $c-M y c$ (Figures $2 \mathrm{c}$ and f). Significant enrichment of p53 at the promoter of $c-M y c$ also occurred in M1 macrophages and this was unaffected by nutlin-3a (Supplementary Figure S2C) coinciding with little $c-M y c$ expression in these cells (Figure 2f). Thus, p53 transcriptionally represses $c-M y c$ gene expression by directly associating with its promoter. Even the modest amount of p53 present in M2-polarized macrophages is sufficient to bind to the $c-M y c$ promoter and limit its transcription, which is consistent with the augmented expression of C-MYC observed in both $\mathrm{p} 53^{-/-}$and $\mathrm{p} 53^{\mathrm{R} 172 \mathrm{H}}$ mutant cells polarized to the $\mathrm{M} 2$ subtype (Figures $4 \mathrm{~b}$ and i).

We propose that p53 suppresses transcription of the $c-M y c$ gene by binding to its promoter region, triggering chromatin remodeling and influencing the expression of a subset of c-MYC-regulated M2 genes.

P53 regulates polarization of M2 macrophages in vivo. The role of $\mathrm{p} 53$ in $\mathrm{M} 2$ polarization in vivo was also determined. Expression of Arg1, Irf4, Retnla (Fizz1) and $c-M y c$ was increased in peritoneal macrophages from $\mathrm{p} 53^{-/-}$ mice administered slow-releasing IL4 (IL4C; Figures 6a-d). IL4C also increased the number of viable peritoneal macrophages to a greater extent in $\mathrm{p} 53^{-1-}$ than wild-type animals (Figures $6 \mathrm{e}$ and $\mathrm{f}$ ). Thus, as in vitro, endogenous p53 regulates IL4-elicited M2 functional phenotype in peritoneal macrophages in vivo.

As M2-polarized macrophages are important for the development of tolerance to LPS, ${ }^{13}$ the effect of nutlin-3a on LPS tolerance in macrophages in vitro and in vivo was studied. Macrophages exposed to a 'challenge' concentration of LPS (M/L) showed increased expression of proinflammatory Tnfa and $1 / 6$ (Figures $7 a$ and $b$ ), which was abolished by prior exposure to a 'tolerance-inducing' concentration of LPS in the presence of the vehicle (dimethylsulfoxide (DMSO) $[(L+D) L]$ ). Nutlin-3a, added during the 'induction' period $[(\mathrm{L}+\mathrm{N}) \mathrm{L}]$, partially restored the expression of Tnfa and I/6 (Figures $7 \mathrm{a}$ and $\mathrm{b}$ ).

Figure 2 Effect of nutlin (Nut)-3a induced p53 activation on gene expression in polarized macrophages. (a) Heatmap of differentially expressed genes between M2-polarized macrophages in the presence or absence of nutlin-3a $(10 \mu \mathrm{M})$. Results show higher (red) or lower (green) expression after drug treatment. Color scale is shown at the bottom, whereby $\log _{2}$ expression values were scaled to range from -2 to +2 . Data are from a single experiment and are representative of three independent samples. (b-e) Expression of Irf4 (b), c-Myc (c), $\operatorname{Arg} 1$ (d) and Retnla (Fizz1; e) in untreated (M0) and M1- and M2-polarized macrophages determined at either 4 or $24 \mathrm{~h}$ of polarization. In some experiments, M2 macrophages were treated with nutlin-3a (10 or $20 \mu \mathrm{M})$. Data show fold change in gene expression by RT-qPCR ( $\Delta \Delta \mathrm{Ct}$ method), and are mean \pm S.D., $n=6$ and are representative of three independent experiments. ${ }^{*} P<0.05$ cf. M2. (f) Expression of C-MYC, IRF4, FIZZ1, ARG1 and p21 proteins in untreated (M0) and polarized (M1 and M2) macrophages as assessed by western blotting and determined at $24 \mathrm{~h}$ of polarization. In some experiments, M2 macrophages were treated with either DMSO or nutlin-3a (10 or $20 \mu \mathrm{M}$ ). Results are from an individual experiment with $\beta$-ACTIN as a loading control and are representative of three independent experiments. See also Supplementary Figure $\mathrm{S} 1$ 
a
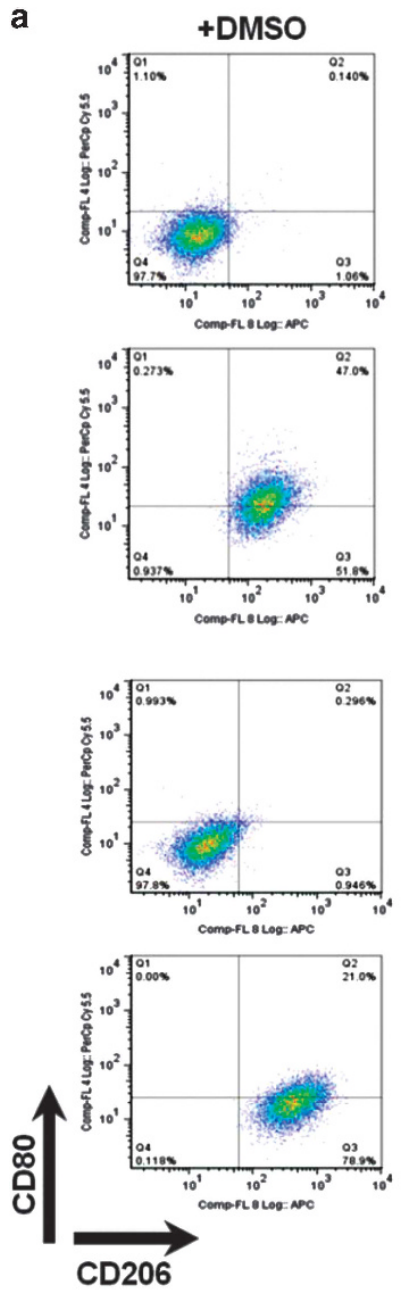
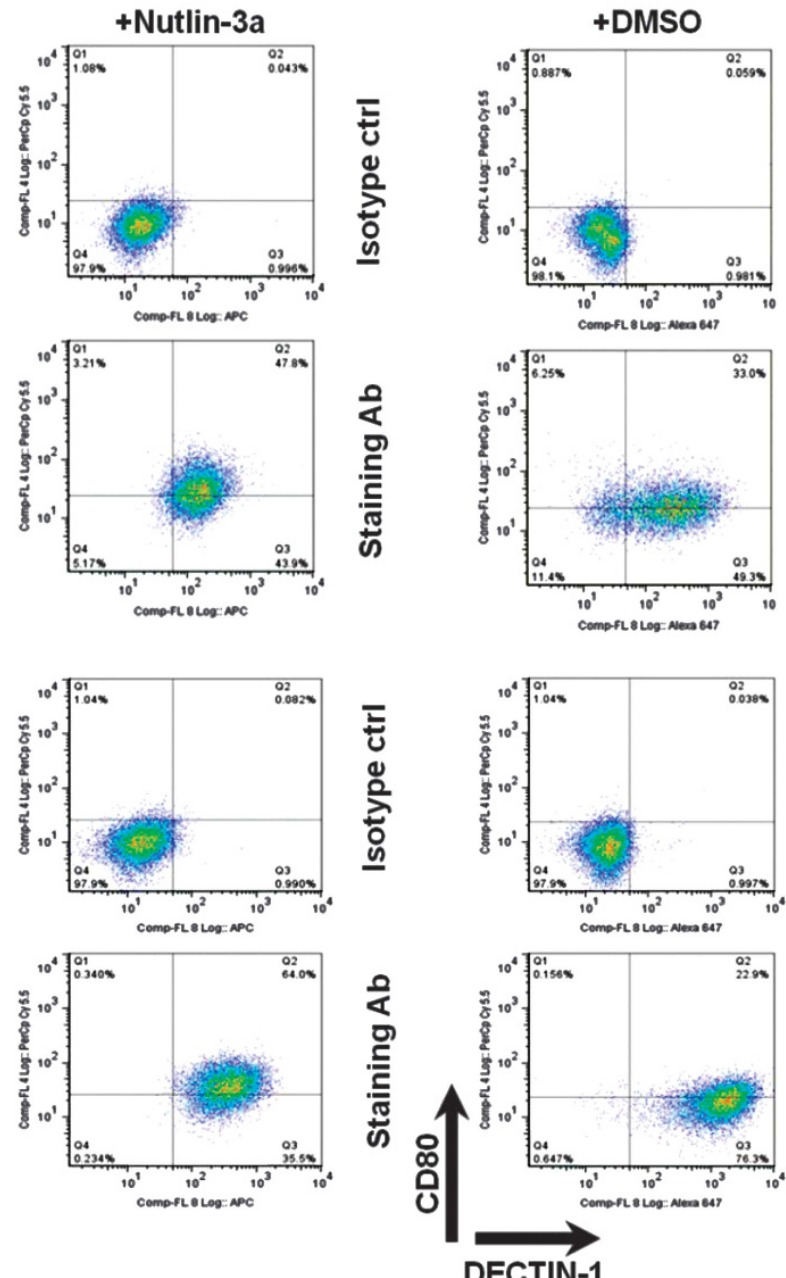

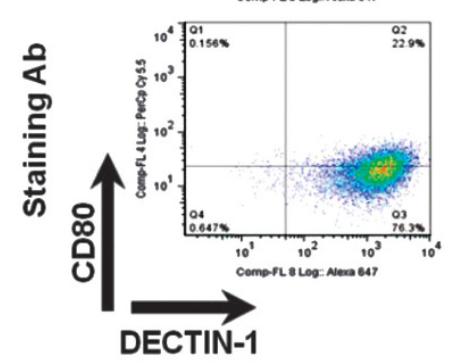

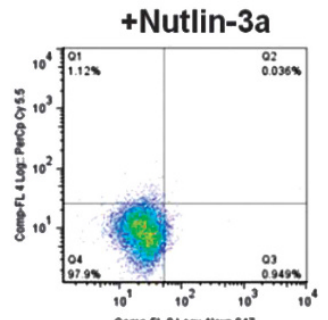

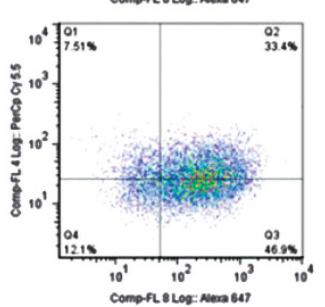

M1

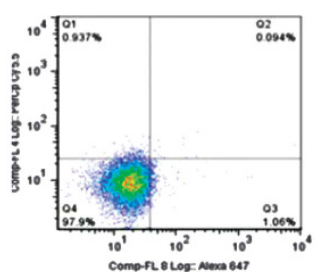

M2
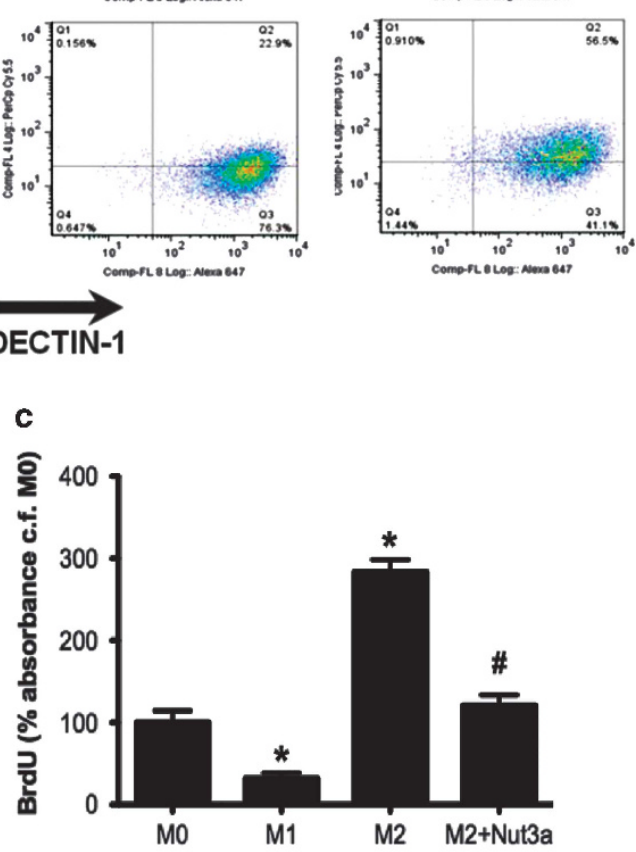

d

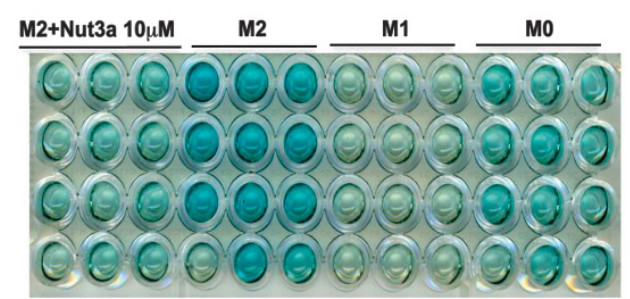


a

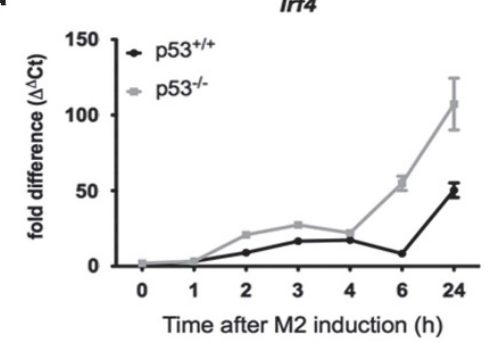

d

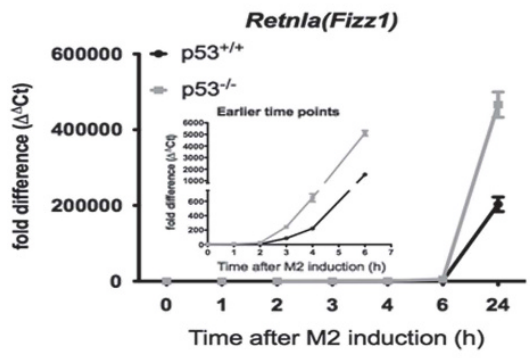

g

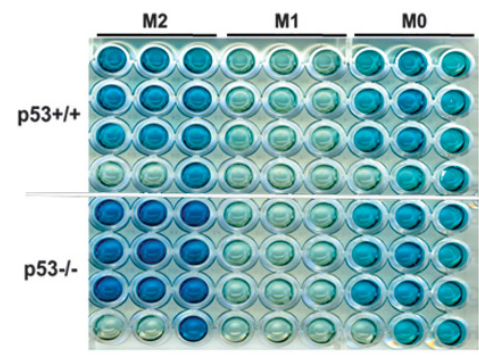

b

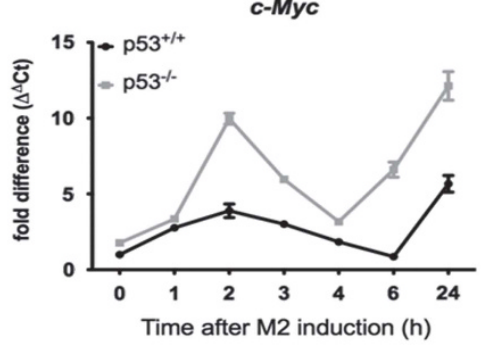

e

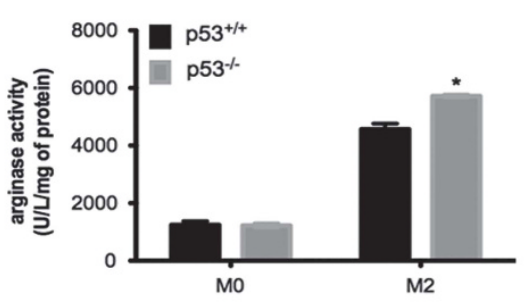

h

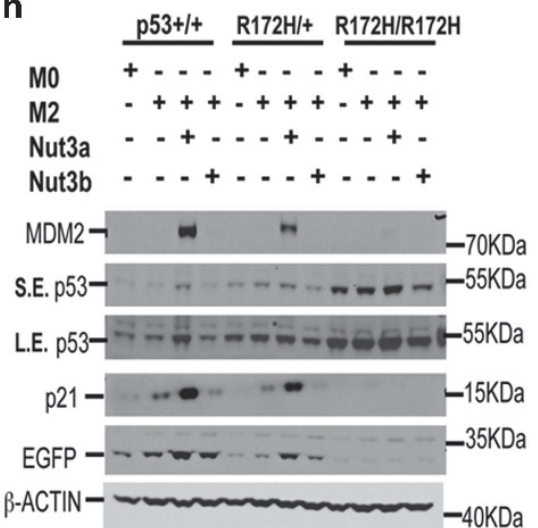

C

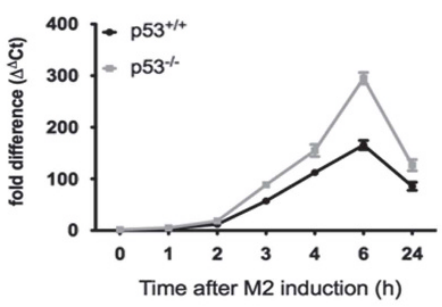

$f$

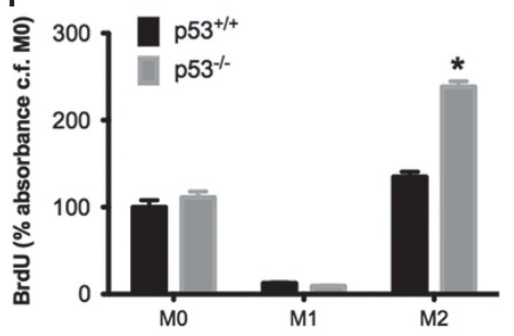

i

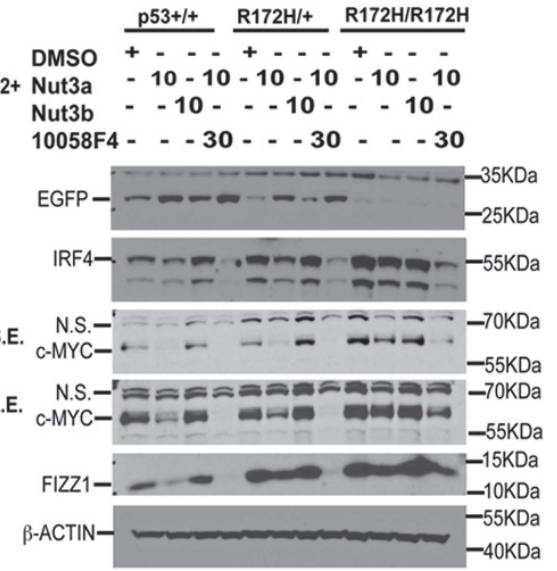

Figure 4 Effect of endogenous p53 activation on M2 gene expression. (a-d) Time-dependent expression of M2 marker genes Irf4 (a), c-Myc (b), Arg1 (c) and Retnla (Fizz1; d) in M2-polarized macrophages derived from $p 53^{+/+}$and $p 53^{-/-}$mice. Data show fold change in gene expression by RT-qPCR $(\Delta \Delta \mathrm{Ct}$ method), and are mean \pm S.D., $n=6$ and are representative of three independent experiments. (e) Arginase catalytic activity determined in M0- or M2-polarized macrophages from p53 ${ }^{+/+}$and $p 53^{-/-}$mice at $24 \mathrm{~h}$ of polarization. Data show enzyme activity expressed as U///mg protein and are mean \pm S.E.M. of three independent experiments. ${ }^{*} P<0.05$ cf. $p 53^{+/+}$. (f and $\mathbf{g}$ ) Measurement of

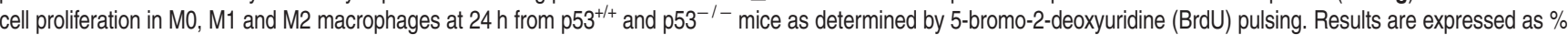
absorbance cf. M0 control in $\mathrm{p} 53^{+/+}$cells after subtraction of results for medium-only controls (f), and show mean \pm S.E.M., $n=3 .{ }^{*} P<0.05$ cf. p53 ${ }^{+/+}$cells. Representative photograph indicating end point colorimetric change of cells is shown in (g). (h) Western blot data showing p53, p21, MDM2 and EGFP protein expression in M0- or M2-polarized macrophages prepared from $p 53^{+/+}, \mathrm{p} 53^{\mathrm{R} 172 \mathrm{H} /+}$ and $\mathrm{p} 53^{\mathrm{R} 172 \mathrm{H} / \mathrm{R} 172 \mathrm{H}}$ mice crossed with $\mathrm{p} 53$ reporter strain in the presence of either nutlin (Nut)-3a (10 $\left.\mu \mathrm{M}\right)$ or nutlin-3b (10 $\left.\mu \mathrm{M}\right)$ for $24 \mathrm{~h} . \beta$-ACTIN was used as a loading control. SE, short exposure; LE, long exposure. Results are representative of three independent experiments. (i) Western blot data showing EGFP, IRF4, c-MYC and FIZZ1 protein expression in M2-polarized macrophages prepared from $\mathrm{p} 53^{+/+}$, $\mathrm{p} 53^{\mathrm{R} 172 \mathrm{H} /+}$ and $\mathrm{p} 53^{\mathrm{R} 172 \mathrm{H} / \mathrm{R} 172 \mathrm{H}}$ mice crossed with $\mathrm{p} 53$ reporter strain in the presence of either nutlin-3a, nutlin-3b (both $10 \mu \mathrm{M})$ or a combination of nutlin-3a $(10 \mu \mathrm{M})$ and the c-MYC inhibitor, $10058 \mathrm{~F} 4(30 \mu \mathrm{M})$, for $24 \mathrm{~h}$. Loading control was $\beta$-ACTIN. Results are representative of two independent experiments. NS, non specific. NS, not significant: See also Supplementary Figures S3 and S4

Figure 3 Effect of nutlin (Nut)-3a induced p53 activation on M2 macrophage phenotype. (a) FACS analysis shows staining of CD80 (M1 marker), CD206 (M2 marker) or DECTIN-1 (M2 marker) in M1- (upper 2 panels) and M2- (lower 2 panels) polarized macrophages exposed to nutlin-3a (10 $\mu \mathrm{M}$ ) or vehicle (DMSO) for $24 \mathrm{~h}$. Panels show dot plot, and representative percentages indicate each subset as a proportion of total $\mathrm{F} 4 / 80^{+} \mathrm{CD} 11 \mathrm{~b}^{+}$macrophages. Results show an individual experiment, which is representative of three independent experiments. (b) Arginase catalytic activity determined in M0- and M2-polarized macrophages exposed to nutlin-3a (10 $\mu \mathrm{M}$ ) or vehicle (DMSO) for $18 \mathrm{~h}$. Data show enzyme activity expressed as U///mg protein and are mean \pm S.E.M., $n=4$. ${ }^{*} P<0.05$, cf. DMSO. (c and d) Measurement of cell proliferation in M0, M1 and M2 macrophages at $24 \mathrm{~h}$ from p53 ${ }^{+/+}$mice as determined by 5-bromo-2-deoxyuridine (BrdU) pulsing. In some cases, M2 macrophages were incubated with nutlin-3a (10 $\mu \mathrm{M}$ ). Results are expressed as \% absorbance cf. M0 control (ctrl) after subtraction of results for medium-only controls (c), and show mean \pm S.E.M., $n=3,{ }^{\star} P<0.05$ cf. M0 and ${ }^{\#} P<0.05$ cf. M2 condition. Representative photograph indicating end point colorimetric change of cells is shown in (d). See also Supplementary Figure S2. Ab, antibody 
a

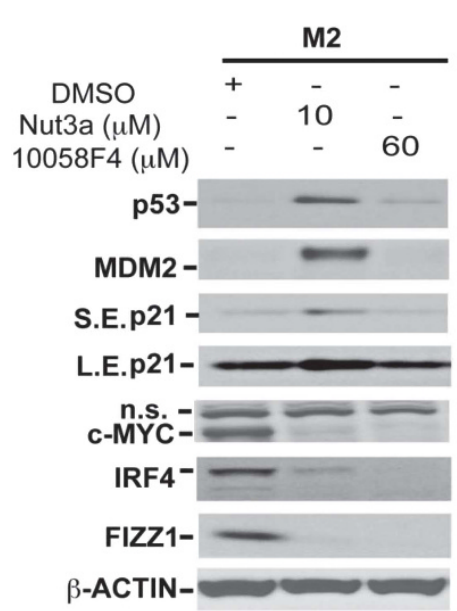

b

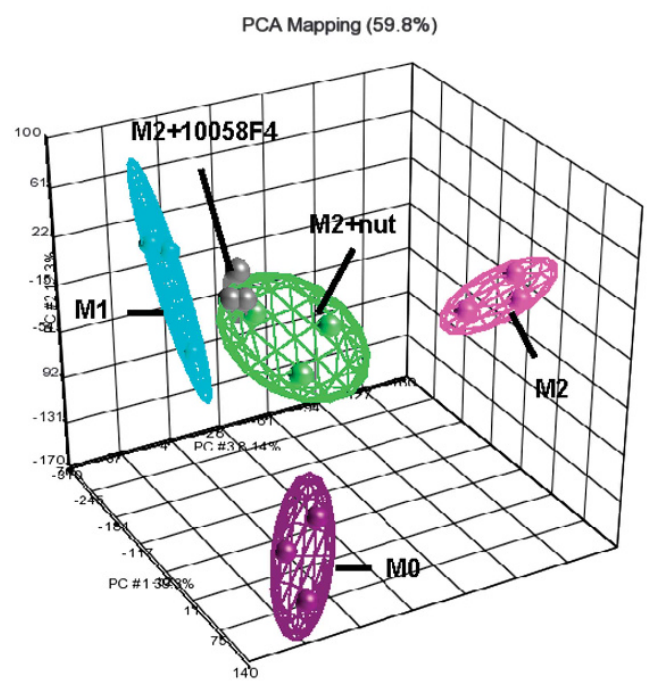

C
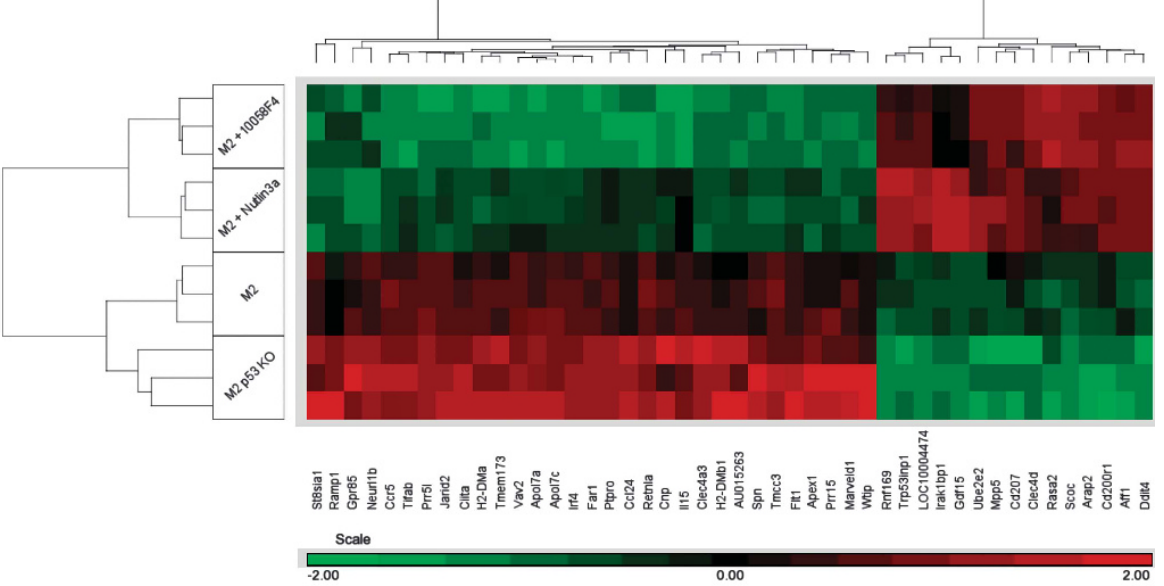

d

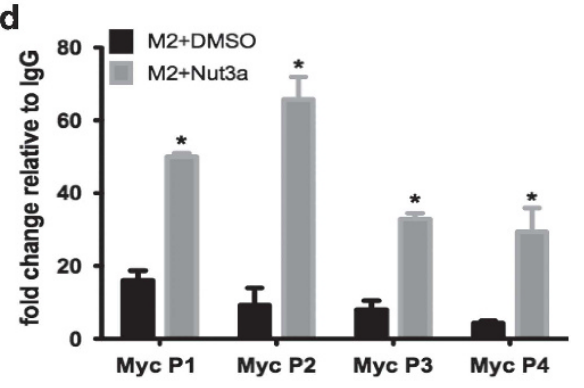

e

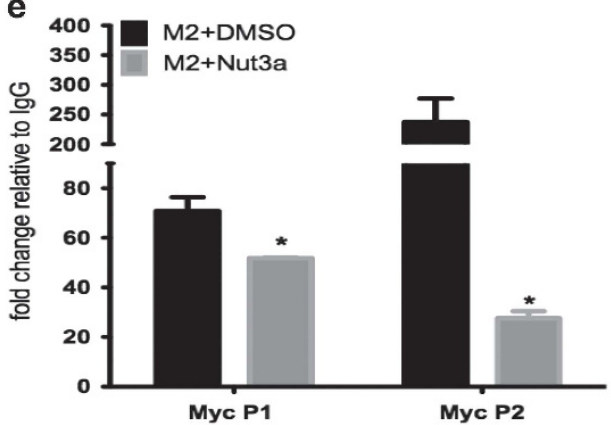

Figure 5 Effect of c-MYC inhibitor on the p53 system and M2 gene expression in M2-polarized macrophages. (a) Western blot data showing the effect of c-MYC inhibitor, 10058F4 (60 $\mu \mathrm{M})$, nutlin (Nut)-3a (10 $\mu \mathrm{M})$ or vehicle (DMSO) on the expression of c-MYC, IRF4, FIZZ1, p53, MDM2 and p21 proteins with $\beta$-ACTIN as a loading control in M2polarizaed macrophages determined at $24 \mathrm{~h}$. Results are representative of three independent experiments. NS, non specific. (b) 3D principal component analysis (PCA) plot of microarray data showing the expression profile of untreated (M0) and polarized (M1 and M2) macrophages incubated for $24 \mathrm{~h}$. In some experiments, M2 macrophages were incubated with either nutlin-3a $(10 \mu \mathrm{M})$ or 10058F4 $(60 \mu \mathrm{M})$. Each dot represents an individual sample and each color represents the treatment type of sample. (c) Heatmap showing differentially expressed genes found in $\mathrm{M} 2$-polarized $\mathrm{p} 53^{+/+}$and $\mathrm{p5}^{-1-}$ macrophages and in M2-polarized p53 $3^{+/+}$macrophages in the presence or absence of nutlin-3a $(10 \mu \mathrm{M})$ or $10058 \mathrm{~F} 4(60 \mu \mathrm{M})$. Results show high gene expression (in red) and low gene expression (in green). Color scale is shown at the bottom, whereby log 2 expression values are scaled to range from -2 to +2 . Data show one microarray experiment using three mice per treatment group. (d) Recruitment of p53 to four different loci of the $c-M y c$ gene promoter in M2-polarized RAW 264.7 macrophages in the presence or absence of nutlin-3a (10 $\mu \mathrm{M}, 2 \mathrm{~h}$ pretreatment) or vehicle (DMSO). Results are assessed as enrichment of the qPCR signal in samples incubated with antibody against p53 compared with an immunoglobulin $\mathrm{G}(\mathrm{lgG})$ control. Data show mean \pm S.E.M. of three independent experiments. ${ }^{*} P<0.05$ cf. M2+DMSO. (e) H3K9ac enrichment at two loci of the $c$-Myc gene promoter in M2-polarized RAW 264.7 macrophages in the presence or absence of nutlin-3a $(10 \mu \mathrm{M}, 2 \mathrm{~h}$ pretreatment) or vehicle (DMSO). Results are assessed as enrichment of the qPCR signal in samples incubated with antibody against acetylated H3K9 compared with an IgG control. Data show mean \pm S.D. of two independent experiments. ${ }^{*} P<0.05$ cf. M2+DMSO. See also Supplementary Figure S5

Tolerance to LPS was associated with reduced p53 activity (Egfp expression) and increased expression of Arg1 and $c-M y c$, which could be reduced by nutlin-3a (Figure 7c). Therefore, activating p53 with nutlin-3a reduced the M2mediated process of LPS tolerance in macrophages.

We then evaluated whether nutlin-3a affected tolerance to LPS in mice in vivo. The timing of LPS and drug injection in these animals is shown in Figure 7d. A 'challenge' dose of LPS

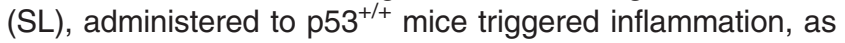
evidenced $6 \mathrm{~h}$ later, by increased plasma TNFa and IL6 (Figures $7 \mathrm{e}$ and $\mathrm{f}$ ), histological signs of alveolar wall thickening, interstitial edema and leukocytic alveolitis (Figure 7g) and increased liver and lung myeloperoxidase (MPO) enzyme activity (Figure 7h). These inflammatory 

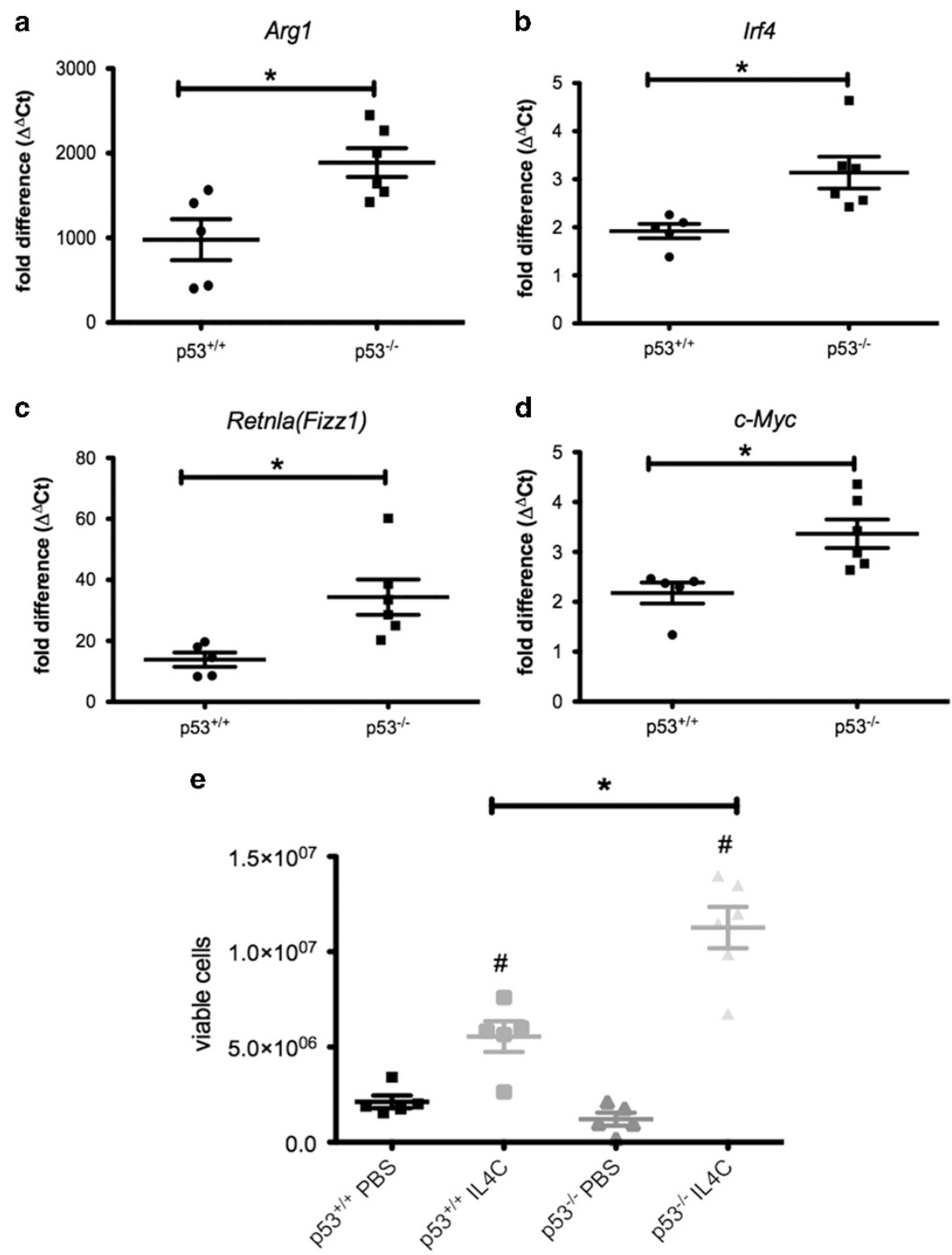

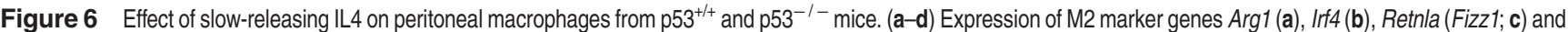

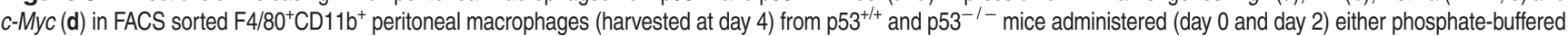
saline (PBS) or IL4C. Data show fold change in gene expression by RT-qPCR ( $\Delta \Delta$ Ct method) cf. PBS-treated p53 ${ }^{\text {t/ }}$ macrophage samples. Individual data for five to six mice per group are presented and are representative of two independent experiments. ${ }^{*} P<0.05 \mathrm{cf}$. IL4C-treated p53 ${ }^{+/+}$macrophage. (e) Number of viable peritoneal macrophages in $\mathrm{p} 53^{+/+}$and $553^{-1-}$ mice administered either PBS or IL4C determined at day 4. Results are from five to six mice per group and are representative of two independent experiments. ${ }^{\#} P<0.05$ cf. PBS-treated macrophages; ${ }^{*} P<0.05$ cf. p53 ${ }^{+/+}$macrophages

indicators were diminished by prior treatment with a lower 'inducing' dose of LPS (LSL), thus confirming the development of LPS tolerance. Nutlin-3a injected after the 'inducing' dose of LPS (LNL) retarded LPS tolerance (Figures 7e-h). In control experiments, nutlin-3a given to $\mathrm{p} 53^{-/-}$mice did not alter plasma TNFa (Supplementary Figure S6A) or IL6 (Supplementary Figure S6B) levels or affect lung histology (Supplementary Figure S6C) in LPS-tolerant animals. We conclude that activation of p53 by nutlin-3a retards LPS tolerance in mice in vivo.

Interestingly, no difference in LPS tolerance was apparent in p53 $3^{-1-}$ cf. $\mathrm{p} 53^{+/+}$mice using this regimen (Supplementary Figures S6A and B), suggesting that loss of p53 does not affect LPS tolerance in vivo. However, the degree of LPS tolerance is reportedly dependent on the timing of LPS injection. ${ }^{14}$ In separate experiments, the 'challenge' dose of LPS was given $6 \mathrm{~h}$ (not $24 \mathrm{~h}$ ) after the 'inducing' LPS dose at which time tolerance was only detected in $\mathrm{p} 53^{-1-}$ animals (Supplementary Figure S7B). Thus, depending on the experimental circumstances, loss of p53 predisposes to the development of LPS tolerance.

Finally, the role of endogenous p53 in LPS-evoked tolerance in macrophages was examined ex vivo. Peritoneal macrophages were isolated from mice $6 \mathrm{~h}$ after an 'inducing' dose of LPS, maintained in culture and then 'challenged' with LPS. Tolerance to LPS was indicated by reduced secretion of TNFa 
and IL6 (Supplementary Figure S7C), diminished expression of Tnfa (Supplementary Figure S7D), II6 (Supplementary Figure S7E), $1 / 12 p 40$ (Supplementary Figure S7F), Cxc/9 (Supplementary Figure S7G) and Cxcl10 (Supplementary Figure $\mathrm{S} 7 \mathrm{H}$ ) and induction of the $\mathrm{M} 2$ genes, $c-M y c$ (Supplementary Figure S7I) and Arg1 (Supplementary Figure $\mathrm{S} 7 \mathrm{H})$. These changes were only apparent in cells from p53 $3^{-1-}$ but not wild-type, mice. We conclude that lack of p53 enhanced LPS tolerance ex vivo.
Overall, these data show that endogenous p53 regulates M2 polarization during LPS tolerance in vitro, ex vivo and in vivo.

\section{Discussion}

M1 polarization is associated with increased p53 expression and activation of downstream genes. P53 inhibits NF- $\mathrm{B}$ (nuclear factor $k \mathrm{~B}$ ) and STAT1 (signal transducer and activator
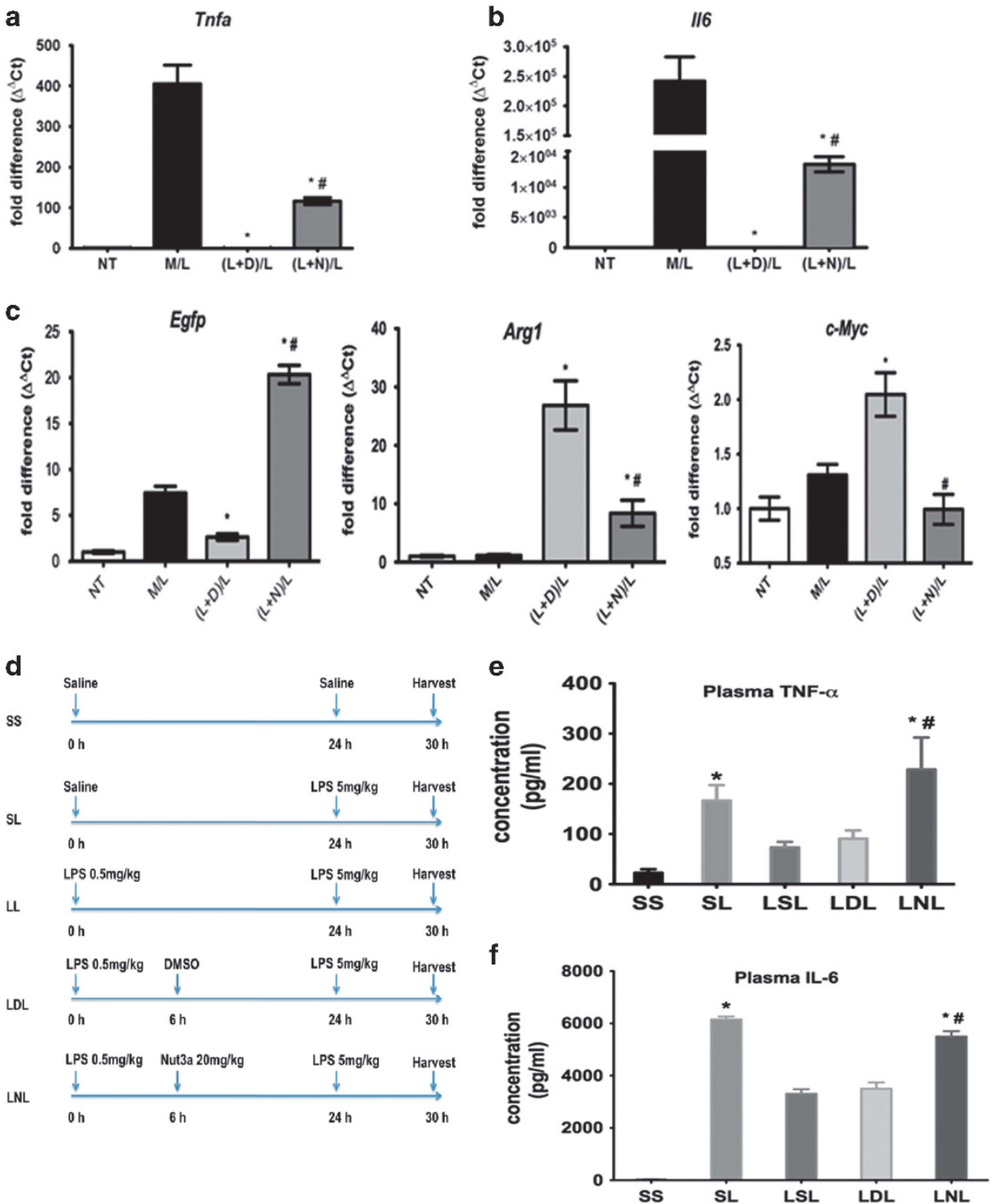

Figure 7 Effect of p53 activation on M2-mediated changes in LPS tolerance in vitro and in vivo. (a-c) Effect of nutlin-3a on LPS-induced tolerance in vitro. Cells were either left untreated (NT) or incubated in medium alone and then treated with LPS (100 ng/ml; M/L). For tolerance experiments, cells were first incubated in medium containing LPS $(100 \mathrm{ng} / \mathrm{ml})$ and DMSO vehicle $[(\mathrm{L}+\mathrm{D}) / \mathrm{L}]$ or nutlin-3a $(10 \mu \mathrm{M} ;(\mathrm{L}+\mathrm{N}) / \mathrm{L})$ and then challenged with LPS $(100 \mathrm{ng} / \mathrm{ml})$ again $24 \mathrm{~h}$ later. Results show expression of Tnfa $(\mathbf{a}), 1 / 6(\mathbf{b})$, Egfp, Arg1 and c-Myc (c) and show fold change in gene expression by RT-qPCR ( $\Delta \Delta \mathrm{Ct}$ method), and are mean \pm S.D., $n=6-8$ and are representative of three independent experiments. ${ }^{*} P<0.05$ (cf. M/L) and ${ }^{\#} P<0.05$ (cf. (L+D)/L). (d) Scheme showing procedure used to evaluate induction of LPS tolerance in vivo. (e and f) Effect of nutlin-3a $(20 \mathrm{mg} / \mathrm{kg}$, intraperitoneally LNL) or DMSO-containing vehicle (LDL) on plasma TNF $\alpha(e)$ and IL6 (f) in LPS-tolerant animals in vivo. Data show mean \pm S.E.M., $n=5-15$. ${ }^{\star} P<0.05$ (cf. SS) and ${ }^{\#} P<0.05$ (cf. LDL). (g) Hematoxylin and eosin sections from lungs of saline-injected control (SS), LPS-challenged (SL), LPS-tolerant (LSL) and LPStolerant mice administered either nutlin-3a (20 mg/kg, intraperitoneally, LNL) or DMSO-containing vehicle (LDL). Results are representative of three independent experiments using at least three mice per group. Magnification, $\times 100$. Scale bar shows $100 \mu \mathrm{m}$. (h) Effect of nutlin-3a ( $20 \mathrm{mg} / \mathrm{kg}$, intraperitoneally) on lung and liver MPO activity from salineinjected control control (SS), LPS-treated (SL) and LPS- tolerant (LSL) mice administered either nutlin-3 (20 mg/kg, intraperitoneally, LNL) or DMSO vehicle (LDL). Data show mean \pm S.E.M., $n=8-12 .{ }^{*} P<0.05$, cf. SL. See also Supplementary Figures $\mathrm{S} 6$ and $\mathrm{S} 7$ 


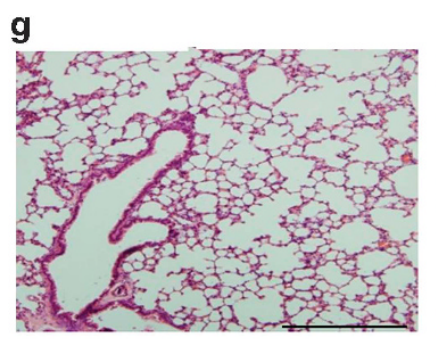

SS

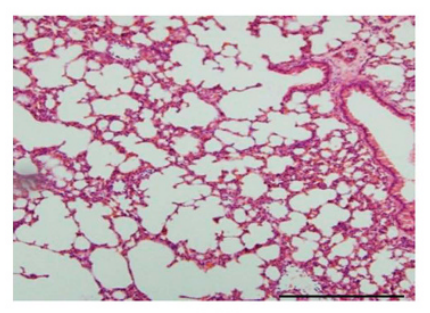

LSL

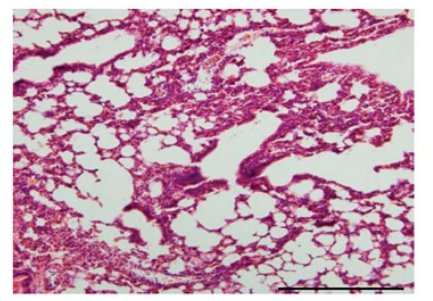

SL

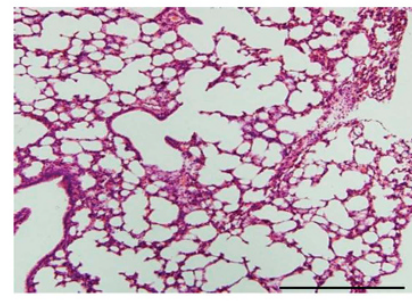

LDL

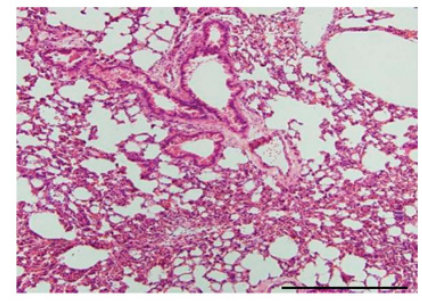

LNL

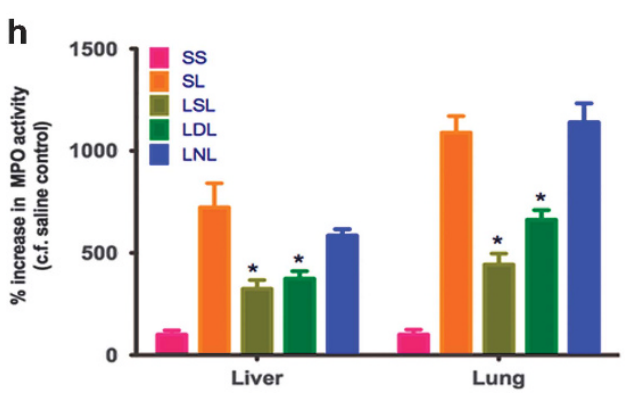

Figure 7 (continued)

of transcription 1) signaling in M1 macrophages, reduces the generation of proinflammatory genes ${ }^{5,15}$ and 'diminishes' the M1 phenotype. The role of p53 in Th2 cytokine-elicited M2 polarization has not previously been described. We show here that upregulated p53 expression and activation of downstream genes is also a feature of M2 polarization, but the extent of the p53 activation is more modest than in M1 cells. IL4 reportedly activates the PI3K/AKT signaling cascade, which is an important feature of M2 polarization. ${ }^{16}$ Our data indicate that activation of PI3K/AKT phosphorylates (and thence activates) MDM2 at Ser166, which then binds to and promotes p53 ubiquitination, thereby limiting p53-mediated transactivation. The critical role of MDM2 in this process is clear from the ability of nutlin-3a, which destabilizes the p53/MDM2 interaction, to potentiate p53 accumulation and transactivation in M2polarized cells.

We propose that p53 has a crucial role in regulating $\mathrm{M} 2$ macrophage polarization. The major consequence of maintaining p53 in a state of low activity in M2-polarized cells is to facilitate M2 gene expression. Albeit modest, the p53 activation that occurs in M2-polarized cells is still important as M2 gene expression was increased both in $\mathrm{p} 53^{-1-}$ macrophages and in cells carrying the structural mutant $\mathrm{p} 53^{\mathrm{R} 172 \mathrm{H}}$. Moreover, 'forcing' activation of p53 in M2-polarized macrophages with nutlin-3a, PM2 or MO11 not only promoted p53 expression and transactivation but also reduced M2 gene expression. The present results show that (i) endogenous p53 activation in M2-polarized macrophages is sufficient to 'blunt' the development of the M2 phenotype, and that (ii) drugs that activate p53 powerfully suppress the M2 phenotype. P53 therefore acts as a 'brake' on M2 macrophage polarization.

Our data suggest that p53 should be added to a list of transcription factors involved in macrophage polarization, which includes STAT1, NF- $K$ B and IRF5 (interferon regulatory factor 5) (important for M1 function), and CREB-C/EBP $\beta$ and JAK-STAT6 (janus kinase-STAT6), which act in concert with PI3K/AKT, IRF4, c-MYC, PPARY (peroxisome proliferatoractivated receptor $\gamma$ ) and KLF4 (Krüppel factor-4) to regulate M2 programming (reviewed by Sica and Mantovani ${ }^{2}$ ). The relative contribution of these pathways to macrophage polarization is poorly defined but the 'master regulators' are likely STAT1/NF-kB for M1 and STAT6 for M2. Within this complex network, p53 has a unique dual role by interacting with NF-KB/STAT1 to regulate M1 polarization and with c-MYC, as we show here, to regulate M2 polarization. Thus, we propose that, in a Th2 cell dominated immune response, STAT6 is required to drive $\mathrm{M} 2$ reprogramming by activating many of the genes (including c-MYC) associated with M2 macrophages. At the same time, IL4 and/or IL13 trigger alternative signaling pathways such as PI3K/AKT, which maintain p53 activity at low levels, thereby facilitating M2 macrophage activation by limiting its transrepressive effect on c-MYC.

We also investigated whether p53 has a part in controlling M2 polarization in vivo. Macrophage expansion can occur by an IL4-driven local proliferation and this is a feature of M2 
macrophage-mediated inflammation. ${ }^{17,18}$ The mechanism underlying this process is not clear, although PI3K/AKT signaling has been implicated. ${ }^{16}$ Here we show that (i) in vitro, the low, basal level of p53 activity in IL4-polarized M2 macrophages is associated with significant cell proliferation and that loss of p53 potentiates, whereas pharmacological activation of p53 impairs M2-mediated proliferation, and (ii) in vivo, IL4 stimulation increased peritoneal macrophage cellularity, which was more marked in the absence of p53. In this manner, p53 is able to regulate M2 macrophage expansion. This, coupled with the ability of p53 to regulate M2 marker expression, raises the possibility that activating macrophage p53 may reduce the density and the protumoral phenotype of M2-like tumor-associated macrophages (TAMs). Indeed, recent reports suggest that p53 may indeed have such a role to play in TAM biology. ${ }^{19,20}$

A low concentration of endotoxin triggers tolerance, which involves the reprogramming of macrophages. ${ }^{21}$ Prior exposure to endotoxin can be an evolutionary advantage because it triggers a selective shutdown of the production of proinflammatory cytokines (from M1 macrophages), which cause tissue damage and preserves the generation of protective antiinflammatory mediators (from M2 macrophages). However, endotoxin tolerance also causes a state of generalized immunosuppression in patients with septic shock, which increases the risk of a secondary, and possibly lethal, infection. Thus, perhaps paradoxically, therapeutic agents that reverse endotoxin tolerance are much sought after.

Recently, enhanced M2 polarization has been noted in endotoxin tolerance ${ }^{13}$ and we therefore used this process as a test of the involvement of p53 in macrophage polarization. Macrophages exposed to LPS on two occasions (i.e. tolerance) showed reduced p53 activity, diminished production of proinflammatory cytokines and augmented expression of M2 marker genes. All of these effects were reversed by nutlin-3a both in isolated macrophages and in mice.

In conclusion, p53 is the first transcription factor reported to suppress M2 macrophage polarization. We propose that manipulation of the p53 system provides an additional approach to study the molecular basis of macrophage plasticity and a new therapeutic target for small molecule p53 activators such as nutlin-3a.

\section{Materials and Methods \\ Collection and culture of cells. Bone marrow-derived macrophages (BMDM) and peritoneal macrophages were obtained from hindlimbs or by peritoneal lavage from mice and cultured as described in Supplementary Methods. Macrophages were incubated in the presence or absence of either LPS and IFN $\gamma$ (Sigma, Singapore) or IL4 and IL13 (PreproTech, Rocky Hill, NJ, USA) to polarize macrophages to the M1 or M2 subtypes, respectively.}

RNA extraction and reverse transcription-qPCR. RNA was prepared from cells using Trizol and reverse transcribed with Superscript II reverse transcriptase (Invitrogen, Singapore). Reverse transcription-quantitative PCR (RT-qPCR) assays, with gene-specific primers spanning introns, were carried out using SsoAdvanced Universal SYBR Green Supermix (Bio-Rad, Singapore) on the Bio-Rad CFX384 real-time PCR detection system. Details of primers are provided in Supplementary Table S3 of Supplementary Methods.

Western blotting. Macrophages were harvested and proteins were extracted using radioimmunoprecipitation buffer. Protein concentration was measured using bicinchoninic acid before western blotting with appropriate antibodies. Details of antibodies are provided in Supplementary Methods.

Arginase activity assay. Arginase catalytic activity was determined colorimetrically by the detection of urea using a commercially available kit (Abnova, Taipei City, Tai Wan).

ChIP assay. ChIP assay was carried out as described in Supplementary Methods or using a ChIP assay kit according to the manufacturer's protocol (Millipore, Singapore).

Live-cell microscopy. Live-cell imaging was performed on an inverted, motorized Nikon $\mathrm{Ti}$ inverted microscope (Singapore) using macrophages maintained in an on-stage incubator $\left(37^{\circ} \mathrm{C}, 5 \% \mathrm{CO}_{2}\right.$ humidified atmosphere).

Endotoxin tolerance experiments. Endotoxin tolerance in vitro was studied in BMDM incubated with LPS (24 h) and then rechallenged with LPS for an additional $4 \mathrm{~h}$. Some cells were exposed to nutlin-3a $(10 \mu \mathrm{M})$. LPS tolerance ex vivo was assessed in peritoneal macrophages cultured from mice that had been injected with LPS $(0.5 \mathrm{mg} / \mathrm{kg})$ and killed $6 \mathrm{~h}$ thereafter. LPS tolerance in vivo was conducted in mice injected with LPS $(0.5 \mathrm{mg} / \mathrm{kg}$, intraperitoneally) followed either 6 or $24 \mathrm{~h}$ later by a second dose of LPS $(5 \mathrm{mg} / \mathrm{kg}$, intraperitoneally). Some animals were given vehicle (DMSO/saline: $3: 7(\mathrm{v} / \mathrm{v}), 1 \mathrm{ml} / \mathrm{kg}$, intraperitoneally) or nutlin-3a $(20 \mathrm{mg} / \mathrm{kg}$, intraperitoneally) $6 \mathrm{~h}$ after the first LPS injection.

Gene expression analysis. Microarray, statistics and bioinformatic analyses are as described in Supplementary Methods. Microarray data can be obtained at the Gene Expression Omnibus database (http://www.ncbi.nlm.nih.gov/geo) with accession code GSE53321.

Statistical analysis. Results show mean \pm S.E.M. or mean \pm S.D. for RTqPCR experiments. Statistical significance of data was determined by Student's $t$-test or one/two-way analysis of variance with post hoc test as appropriate using GraphPad Prism 5 software (La Jolla, CA, USA). $P<0.05$ was taken to indicate statistical significance.

\section{Conflict of Interest}

The authors declare no conflict of interest.

Acknowledgements. This work was supported by grants from the Agency for Science, Technology and Research Singapore (A*STAR) to LL, DPL, FG and AM and from National University of Singapore to PKM. We thank Biopolis Shared Facility Microarray Service and Poh Cheang Chiam for the technical support.

\section{Author contributions}

$\mathrm{LL}$ and DPL conceived the experiments. LL, DPL and PKM wrote the paper. LL, DSWN, WM, FFA, SAR, VKR, SCL, FL and MTP performed experiments. LL, DSWN, WM, VKR, SCL, FL, JSYL, GDW, AM, RT, FG, CGL and PKM analyzed data. AMG provided some of the experimental animals and helped to edit the paper.

1. Murray PJ, Wynn TA. Protective and pathogenic functions of macrophage subsets. Nat Rev Immunol 2011; 11: 723-737.

2. Sica A, Mantovani A. Macrophage plasticity and polarization: in vivo veritas. J Clin Invest 2012; 122: 787-795.

3. Vazquez A, Bond EE, Levine AJ, Bond GL. The genetics of the p53 pathway, apoptosis and cancer therapy. Nat Rev Drug Discov 2008; 7: 979-987.

4. Cook J, Hagemann T. Tumour-associated macrophages and cancer. Curr Opin Pharmacol 2013: 13: 595-601.

5. Liu G, Park YJ, Tsuruta Y, Lorne E, Abraham E. P53 attenuates lipopolysaccharide-induced NF-kappaB activation and acute lung injury. J Immunol 2009; 182: 5063-5071.

6. Komarova EA, Krivokrysenko V, Wang K, Neznanov N, Chernov MV, Komarov PG et al. P53 is a suppressor of inflammatory response in mice. FASEB $J$ 2005; 19: 1030-1032.

7. Elsawy KM, Verma CS, Lane DP, Caves LS. On the origin of the stereoselective affinity of nutlin-3 geometrical isomers for the MDM2 protein. Cell Cycle 2013; 12: 3727-3735.

8. Wei SJ, Joseph T, Chee S, Li L, Yurlova L, Zolghadr K et al. Inhibition of nutlin-resistant HDM2 mutants by stapled peptides. PLOS One 2013; 8: e81068.

9. Vousden KH, Lu X. Live or let die: the cell's response to p53. Nat Rev Cancer 2002; 2 594-604. 
10. Pello OM, Chèvre R, Laoui D, De Juan A, Lolo F, Andrés-Manzano MJ et al. In vivo inhibition of c-MYC in myeloid cells impairs tumor-associated macrophage maturation and pro-tumoral activities. PLoS One 2012; 7: e45399.

11. Pello OM, De Pizzol M, Mirolo M, Soucek L, Zammataro L, Amabile A et al. Role of c-myc in alternative activation of human macrophages and tumor-associated macrophage biology. Blood 2012; 119: 411-421.

12. Beyer M, Mallmann MR, Xue J, Staratschek-Jox A, Vorholt D, Krebs W et al. High-resolution transcriptome of human macrophages. PLoS One 2012; 7: e45466.

13. Pena OM, Pistolic J, Raj D, Fjell CD, Hancock RE. Endotoxin tolerance represents a distinctive state of alternative polarization (M2) in human mononuclear cells. J Immunol 2011; 186: 7243-7254.

14. Nomura F, Akashi S, Sakao Y, Sato S, Kawai T, Matsumoto M et al. Cutting edge: endotoxin tolerance in mouse peritoneal macrophages correlates with down-regulation of surface tolllike receptor 4 expression. J Immunol 2000; 164: 3476-3479.

15. Zheng SJ, Lamhamedi-Cherradi SE, Wang P, Xu L, Chen YH. Tumor suppressor p53 inhibits autoimmune inflammation and macrophage function. Diabetes 2005; 54: 1423-1428.
16. Rückerl D, Jenkins SJ, Laqtom NN, Gallagher IJ, Sutherland TE, Duncan S et al. Induction of IL-4R $\alpha$-dependent microRNAs identifies PI3K/Akt signalling as essential for IL-4-driven murine macrophage proliferation in vivo. Blood 2012; 120: 2307-2316.

17. Jenkins SJ, Ruckerl D, Cook PC, Jones LH, Finkelman FD, van Rooijen $\mathrm{N}$ et al. Local macrophage proliferation, rather than recruitment from the blood, is a signature of TH2 inflammation. Science 2011; 332: 1284-1288.

18. Jenkins SJ, Ruckerl D, Thomas GD, Hewitson JP, Duncan S, Brombacher F et al. IL-4 directly signals tissue-resident macrophages to proliferate beyond homeostatic levels controlled by CSF-1. J Exp Med 2013; 210: 2477-2491.

19. Lowe JM, Menendez D, Bushel PR, Shatz M, Kirk EL, Troester MA et al. P53 and NF-кB coregulate proinflammatory gene responses in human macrophages. Cancer Res 2014; 74: 2182-2192.

20. Lujambio A, Akkari L, Simon J, Grace D, Tschaharganeh DF, Bolden JE et al. Non-cellautonomous tumor suppression by p53. Cell 2013; 153: 449-460.

21. West MA, Heagy W. Endotoxin tolerance: a review. Crit Care Med 2002; 30(Suppl): S64-S73.

Supplementary Information accompanies this paper on Cell Death and Differentiation website (http://www.nature.com/cdd) 